\title{
Doctors dissatisfied with medical careers at high risk of burnout
}

Cite as: CMAJ 2019 November 25;191:E1318. doi: 10.1503/cmaj.1095828

Posted on cmajnews.com on November 4, 2019.

W orkplace stressors are stronger predictors of burnout in physicians than self-care habits or lack thereof, according to a Canadian Medical Association (CMA) survey.

Last year, the association released results of a survey of 2547 physicians and 400 trainee doctors (response rate of $8.5 \%$ of 34,517 CMA members contacted). Nearly a third of respondents reported high levels of burnout. More than a third of participants screened positive for depression and nearly $10 \%$ reported suicidal thoughts in the previous year.

Until recently, most institutions' efforts to tackle these problems have focused on improving resilience through advice on diet, exercise, mindfulness and other individual strategies. According to the CMA, however, these results suggest that a "greater emphasis on reducing occupational stressors within the practice environment is needed."

In a new regression analysis of the survey data, career dissatisfaction was the strongest predictor of burnout, depression and suicidal thoughts, affecting more than a tenth of doctors surveyed. Doctors who reported dissatisfaction with their careers were about seven times more likely to experience burnout, and about four times more likely to report low social, psychological and emotional well-being.

High presenteeism was another strong predictor of poor psychological outcomes, affecting $20 \%$ of survey respondents. Physicians who worked while sick or distressed five or more times in the previous year were about three times more likely to report burnout, depression and suicidal thoughts. Other occupational predictors of burnout included poor work-life integration, low collegiality and

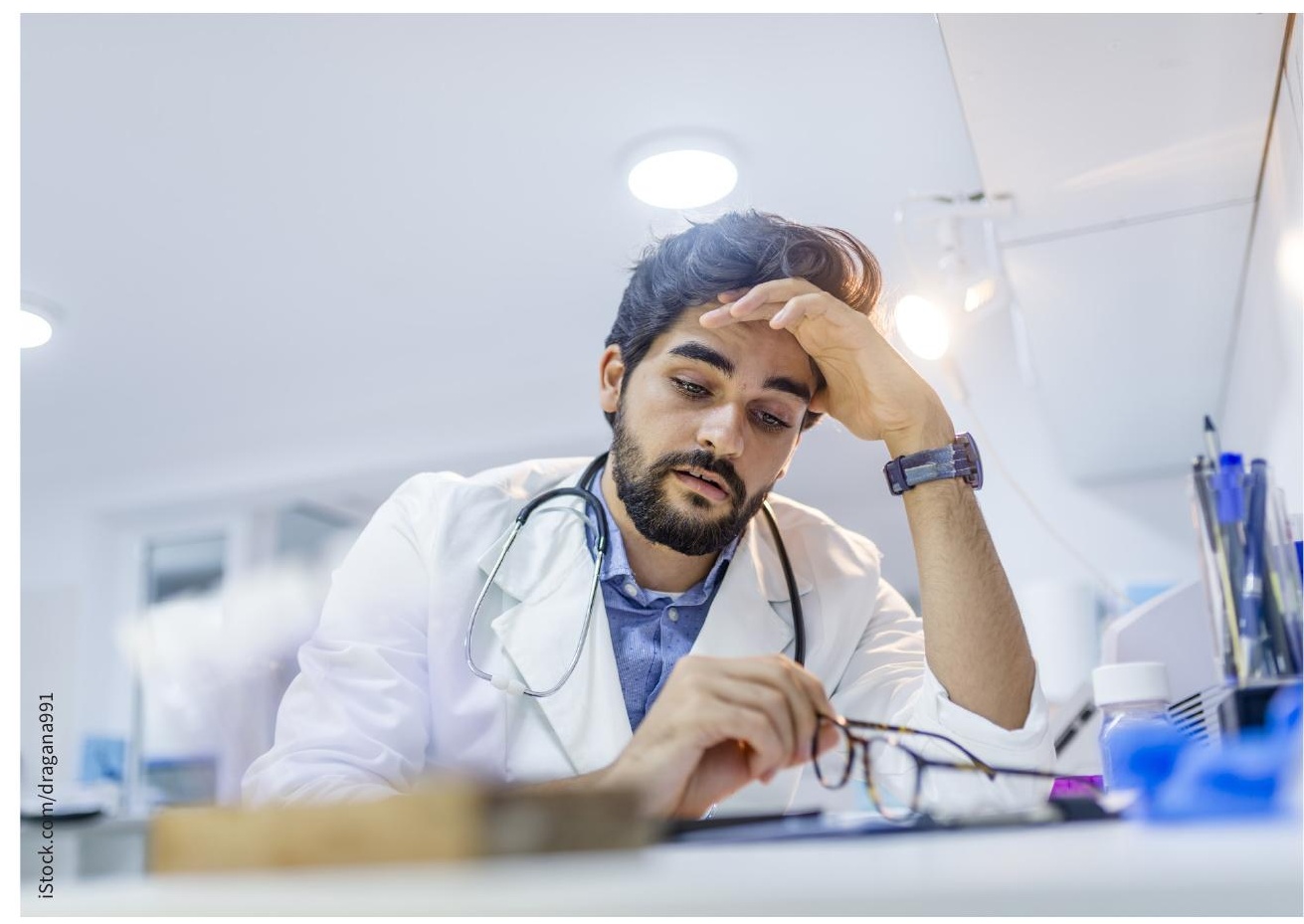

Workplace stressors are strong predictors of burnout and depression among physicians.

dissatisfaction with efficiency and resources in the workplace.

By comparison, lifestyle factors like diet, exercise, having a family doctor, and drug and alcohol use were less strongly predictive of negative psychological outcomes. Doctors who reported unhealthy diets $(22 \%)$ or binge drinking $(7 \%)$ were 1.5 times more likely to experience burnout. Those who reported insufficient physical activity (48\%) were more likely to report low resilience, or difficulty recovering from setbacks.

The CMA survey also showed that the health risks doctors faced differed depending on their gender and career stage. Both women and trainee doctors were more likely to report burnout, career dissatisfaction and presenteeism. Female doctors were 1.3 times more likely to report insufficient physical activity and dissatisfaction with workplace efficiency. Trainees were more likely to use stimulants and eat poorly. Male doctors were less likely to experience burnout than female peers but were also less likely to have a personal doctor and more than twice as likely to smoke and binge drink.

According to CMA President Dr. Sandy Buchman, these results highlight areas to target to improve physician health and wellness. "A healthy workforce is a shared responsibility. We all have a role to play because, ultimately, it's about creating the right environment for physicians to do what they do best: deliver patient care."

Lauren Vogel, CMAJ 